\title{
Floating car data system enforcement through vehicle to vehicle communications
}

\author{
Lan Lin, Tatsuaki Osafune, Massimiliano Lenardi \\ Hitachi Europe SAS. - Hitachi Sophia Antipolis Lab. \\ Immeuble Le Thélème, 1503 Route des Dolines \\ 06560 Valbonne France \\ Email : Lan.Lin@hitachi-eu.com
}

\begin{abstract}
The paper presents a new application enabled by Vehicle-to-Vehicle (V2V) communication systems; our target is to combine V2V with Floating Car Data (FCD) systems and merge advantages of both: the V2V application warnings to enhance the FCD traffic information precision as well as the information availability; inversely, V2V communication can also benefit from the FCD system and offer some large-scale road network traffic information. We present some architecture modifications which are needed to realize this new application, both in terms of in-vehicle components as well as the networking requirements. The proposed approach is to be built upon the existing infrastructure of FCD system to lower the implementation cost.
\end{abstract}

\section{INTRODUCTION}

Vehicle-to-Vehicle (V2V) and Vehicle-to-Infrastructure (V2I) communications have become, since several years, one of the main R\&D trend in ITS field at worldwide scale. Its amazing capability on improving the active safety has attracted high attention. VSC (Vehicle Safety Communications by DSRC) project in USA, ASV (Advanced Safety Vehicles) in Japan and C2CCC (Car-to-Car Communication Consortium) in Europe are the main activities focusing on this field, both in terms of $R \& D$ and standardization efforts. Nevertheless, the market introduction strategy meets a big challenge for this new technology because a certain level of penetration rate is necessary to obtain significant safety gains. In order to increase the penetration rate, some non-safety V2X (e.g. vehicle to home, vehicle to enterprise) enabled applications are taken into consideration for near-term or mid-term implementation such as the infotainment and traffic management efficiency. A wide range of information such as position, speed, safety warnings are available or detected over the vehicular short-range network. The integration of V2V and Floating Car Data (FCD) systems is one potential efficient traffic management application which would be able to utilize short range network messages and extend them a large geographic range if necessary, thanks to the public broadcast media.

The objective of this paper is to describe a FCD enforcement method enabled by $\mathrm{V} 2 \mathrm{~V}$ communications (V2VC). Our intention is to combine FCD and V2VC without deeply modifying them, in order to decrease the investment cost. Both FCD system and V2V systems will benefit from this combination. (i) By using the up-link channel of FCD system (e.g. GPRS or 3G mobile network) to transmit the information available at V2V systems, FCD system users will benefit from some useful local warnings detected by $\mathrm{V} 2 \mathrm{~V}$ systems. (ii) Some large-scale traffic information from the
FCD operation center can be also integrated as a new V2V application.

Simplifying, the V2V equipped vehicles serve as mobile sensors, providing its own floating data to a FCD equipped vehicle inside its communication range; the latter serves as a temporal FCD data collector from the vehicles nearby. Some preliminary data treatment is processed in this collector before sending the results out to the operating centre for further traffic analysis. This approach allows the system to have a higher number of available floating vehicles and to improve the data reliability. A low-cost implementation on the infrastructure side can also be expected - the system is built on the existing communication infrastructure used for data transmission to and from the operation center.

The present paper is divided into five parts, part II is the introduction of some background information; part III will be dedicated to the detailed explanation of the application scenario and in-system architecture, in part VI, a conceptual communication network and some specific requirements will be presented. Finally, conclusions and future work are given in part $\mathrm{V}$.

\section{BACKGROUND}

\section{A. Prior Arts}

One FCD system enabled by V2VC was proposed within the German FleetNET project. A totally decentralized V2V communication approach was chosen. After collecting floating data from the vehicles inside the communication range, a traffic situation analysis is carried out by in-vehicle processing to extract the traffic information, e.g. traffic jams. These information results will then be sent to $20-50 \mathrm{~km}$ distance road network coverage, again through the $\mathrm{V} 2 \mathrm{~V}$ network for the dynamic rerouting calculation in navigation system. This approach was evaluated through simulation. The results showed that, with a $2 \% \mathrm{~V} 2 \mathrm{~V}$ system penetration rate, a transmission delay of about 30 minutes is needed for the transmission of the traffic information. Compared to the conventional centralized FCD system, some constraints exist for this approach:

1) Reduced reliability of the traffic information obtained by in-vehicle processors since V2V communications are targeted at local level.

2) Reduced floating vehicles numbers: the number of the floating car is much less than the centralized FCD system, which might have an impact on the analysis precision.

3) From communication system point of view, large 
transmission bandwidth and a long communication range are required in order to collect enough number of the floating data.

4) From the in-vehicle processor point of view, a high work load is needed for the on board computer in the collector vehicle for the big amount of data. This requirement implies a higher implementation cost on the vehicles.

We think that a total decentralized approach is less realistic for the FCD traffic information system, because a high number of the FCD vehicles is needed to have a precise traffic information extraction (e.g. the length and the location of traffic jams), while the $\mathrm{V} 2 \mathrm{~V}$ system has a quite limited communication bandwidth.

\section{B. FCD for traffic information}

Since its appearance on the market, Floating Car Data as a tool for traffic information collection has proved its big benefits for the road operators and the drivers [5]. The floating data collected from the vehicles or from the roadside sensors, such as position, speed, and directionality, are sent to the operation center for the traffic information analysis. A time window is often used in the algorithm in order to include some short term historical floating data in order to enrich the reliability of the traffic information. At the centre, probe car data is analyzed through specific algorithms, the main outputs include the average speed, the congestion travel time, the traffic jam position, traffic prediction, etc. Some other traffic related information such as weather, traffic management information (e.g. road construction, road closing, etc.) can be also collected from the road operators and broadcasted together with analysis results to the end users through different media. But in the real world implementation, the ownerships of data resources and sensors can be different. For example, the induction coils data can belong to the police, while the mobile phone data is under the control of the telecom operators. Organizational and political reasons may then become the main obstacles to collect the necessary floating data.

Nowadays, two main types of vehicle-based floating data systems are used: taxi/bus FCD and mobile phone FCD systems, both have reached some business success in different cities and countries such as in Germany, in Beijing and in Paris. But at least two constrains remain:

1) Specific mobility pattern for taxis and buses as collective or sharing vehicle services have some negative impacts on the analysis precision. These special patterns include dedicated stops and lanes, too low presence in secondary roads, etc.

2) Difficulty of the map matching for the mobile phone system. Mobile terminals tracking is often used in this kind of systems, where a large amount of the data treatment and a complicated algorithm are needed at the center side for the matching of a certain cellular phone into the moving vehicles. The agreement between the mobile operators and traffic management operators is also needed to implement the system.

3) Floating data sent to the operation center is raw data. After the matching, a lot of data have to be abandoned or considered as useless or imprecise at the operation centre. This is represented as a low ratio of the useful amount of collected data and means the uneconomic consumption of the communication channel.

In this paper, we aim to improve the performance of the vehicle-based FCD systems. The combination of itself with V2V networks can help to resolve the listed shortcomings.

First of all, V2V systems are mainly targeting to the private passenger vehicles. By including these vehicles as the floating vehicles, the mobility patterns will be more realistic.

Second of all, some preliminary data treatment especially on the position data at the vehicle side will improve the position data precision. This first-round analysis at the decentralized way may help to improve the precision of map matching at the centre, and consume more economically the up-link communication channel.

\section{SYSTEM ARCHITECTURE}

A wide variety of the information is available at the $\mathrm{V} 2 \mathrm{~V}$ communications system, where vehicles are considered as the extended sensors.

In European WILLWARN project, obstacles including accidental vehicles, static obstacles, construction sites, traffic jams can be detected, and the corresponding warning messages are generated and broadcasted inside the vehicular short-range network. Apart from these warning messages, vehicles exchange their position, speed information among each other for some other safety applications e.g. the cooperative driving.

By denoting the vehicles equipped with FCD equipments as $F C D$-Vehicles, those equipped with $\mathrm{V} 2 \mathrm{~V}$ systems as $\mathrm{V} 2 \mathrm{~V}$ Vehicles, and those equipped with both systems as mastervehicles, the objectives of linking $\mathrm{V} 2 \mathrm{~V}$ and FCD systems are the following:

1) To increase the floating vehicle numbers: besides the FCD-vehicles, the V2V-vehicles may be able to send their own floating data to the master-vehicles, so the number of the floating vehicles is then increased.

2) To improve the floating data reliability: a preliminary data analysis is processed at the master-vehicle. (i) The data that can be considered with a high probability to be wrong data is discarded before any analysis or to be sent to the operating center. (ii) In many $\mathrm{V} 2 \mathrm{~V}$ applications, the position data used is extrapolated from the vehicles nearby or other data. This position data is more precise or matched than the raw ones. (iii) Moreover, more floating vehicles can also help to improve the analysis precision.

3) To enrich the information: warning messages available through V2V systems can be some useful traffic information that may not be able to be detected through macroscopic FCD analysis.

\section{A. Overview}

Fig. 1 shows the scenario of the system, with the example of "Traffic Jam Detection" application. No road side unit for 
short range communications is used, since our primary objective is to use the existing FCD up- and down-link infrastructure (in many case through cellular phone network) for data transmission to lower the cost of the system implementation. But we do not discard the possibility of using road side units in our architecture once they become available, since we can imagine that a road side unit should use a communication unit similar to the $\mathrm{V} 2 \mathrm{~V}$ ones, by considering it as a static node.

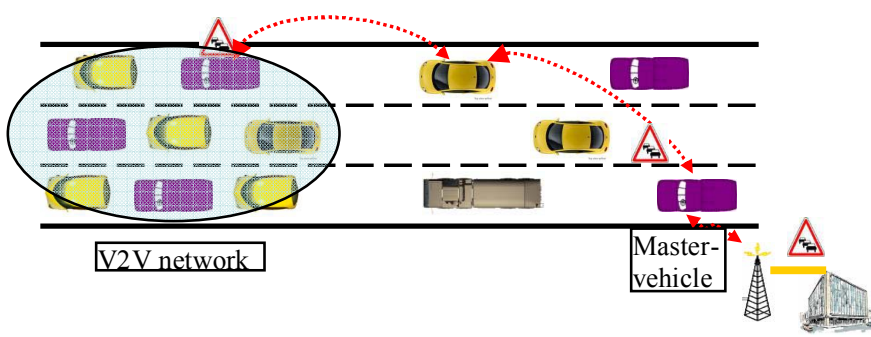

Fig. 1 System scenario and V2V traffic jam detection application

A master-vehicle sends out periodic messages to notify its presence and availability, and sets itself as the head of a cluster network around. A self configuration process is then used in order to assign a network address to the $\mathrm{V} 2 \mathrm{~V}$ Vehicles in this cluster network and any V2V-Vehicle entering the range. These $\mathrm{V} 2 \mathrm{~V}$-vehicles will then continuously send to the master-vehicle their own floating data, such as position, time stamp, speed and sensor data. A unicast routing protocol like MOPR (Movement Prediction based Routing, [6]) is used for this transmission.

As mentioned, two types of traffic jam detection methods are possible:

1) At operating centers through FCD analysis algorithms: the method is the same as the classic FCD systems, where the traffic jam is extracted by floating data analysis algorithms at the operation center. A macroscopic algorithm is used for that purpose.

2) Through $V 2 V$ communication applications. Local traffic jam, for example, can be detected by V2V communications by vehicle tracking and driving dynamics. In fact, numerous other safety or traffic efficiency warnings can be generated through V2V communications e.g. accident, urgent brake etc. These warnings can be also included into the FCD data packet. These two approaches are illustrated in Fig. 1.

Master-vehicles are keeping a list of other vehicles data in their memory space with the format of Table 1. A time window is set to store the data from the same vehicle at different time stamps in the memory: short-term historical data are useful to improve the analysis precision and should be also included in the data analysis process. An expiration period should be set in the table to delete obsolete data. This expiration period should be no longer than the transmission time interval of the probe data to the operation center, this interval can vary from several seconds to several minutes, according to the system implementation.

The collected FCD data of all the V2V-Vehicles and
Master-vehicle itself are sent to the operation center, the analysis results (e.g. traffic jams and their positions, average speed, travel time etc.) will then be sent through broadcasting media to the vehicles at a large-scale geographic area, this information can also be extended from the receiving mastervehicle to the nearby $\mathrm{V} 2 \mathrm{~V}$ vehicles in communication range, i.e. using I2V communications as well.

An example of the master-vehicle table is shown in Tab. 1.

\begin{tabular}{|c|c|c|c|c|c|c|}
\hline $\begin{array}{c}\text { Vehicle } \\
\text { ID }\end{array}$ & $\begin{array}{c}\text { Time } \\
\text { stamp }\end{array}$ & $\begin{array}{c}\text { Speed } \\
(\mathrm{Km} / \mathrm{h})\end{array}$ & $\begin{array}{c}\text { Projected } \\
\text { Position }\end{array}$ & $\begin{array}{c}\text { Time } \\
\text { to } \\
\text { expire } \\
\text { (count })\end{array}$ & Warning & Direction \\
\hline A & $\begin{array}{c}\text { Hh:mm: } \\
\text { ss.ss }\end{array}$ & 30 & Xx:xx:xx & 3 & $\begin{array}{c}\text { Wet } \\
\text { road }\end{array}$ & $\begin{array}{c}\text { Down } \\
\text { stream }\end{array}$ \\
\hline A & $\begin{array}{c}\text { Hh:mm: } \\
\text { ss.ss }\end{array}$ & 25 & Xx:xx:xx & 5 & $\begin{array}{c}\text { Wet } \\
\text { raod }\end{array}$ & $\begin{array}{c}\text { Down } \\
\text { stream }\end{array}$ \\
\hline B & $\begin{array}{c}\text { Hh:mm: } \\
\text { ss.ss }\end{array}$ & 20 & Xx:xx:xx & 7 & $\begin{array}{c}\text { End of } \\
\text { jam }\end{array}$ & $\begin{array}{c}\text { Up } \\
\text { stream }\end{array}$ \\
\hline$\ldots$ & $\ldots$ & $\ldots$ & $\ldots$ & $\ldots$ & $\ldots$ & $\ldots$ \\
\hline
\end{tabular}

Table 1: nearby vehicle list

Vehicle ID: Temporal vehicle identity in the cluster network;

Time stamp: Received time of the data packet, hour, minute, second;

Velocity: Speed and direction of the vehicle at that time stamp

Projected position: Extrapolated GPS position from the nearby vehicles or other position data, this projected position is defined in VSCC project especially for the cooperative applications.

Time to expire: Count down of time stamp units to which the data is discarded because of the expiration.

Warning: Warning messages detected or generated through V2V systems.

Directionality: Driving direction of the vehicle.

\section{B. System components}

In our FCD-V2V system, there is no significant modification for the vehicle to operation center communication infrastructure. Nevertheless, some updates of the data analysis algorithm at the operation center are enough to make it adapted to FCD data packets that include more information. That is to say, no hardware investment is needed at center and the communication infrastructure.

Concerning the vehicles, the biggest modification is for the master-vehicles. They need to be equipped both FCD and V2V On-Board Units (OBUs): both of them can be available for the sake of the implementation at each system. But some interfaces are needed to integrate these two OBUs for the data sharing and transmission.

Fig. 2 shows the block diagram at vehicles. It shows the main components and sub-systems needed for the mastervehicle operations; the dotted line excludes the component (FCD Radio Unit) which is not necessary at the V2V-vehicle.

The information transmitted by vehicles includes their position, velocity, directionality as well as the warning messages forwarded by $\mathrm{V} 2 \mathrm{~V}$ networks. These data are transferred into the master-vehicle's central processor, together with its own position and sensor data, and analyzed. 
The block diagram of the processor module is detailed in the Fig. 3.
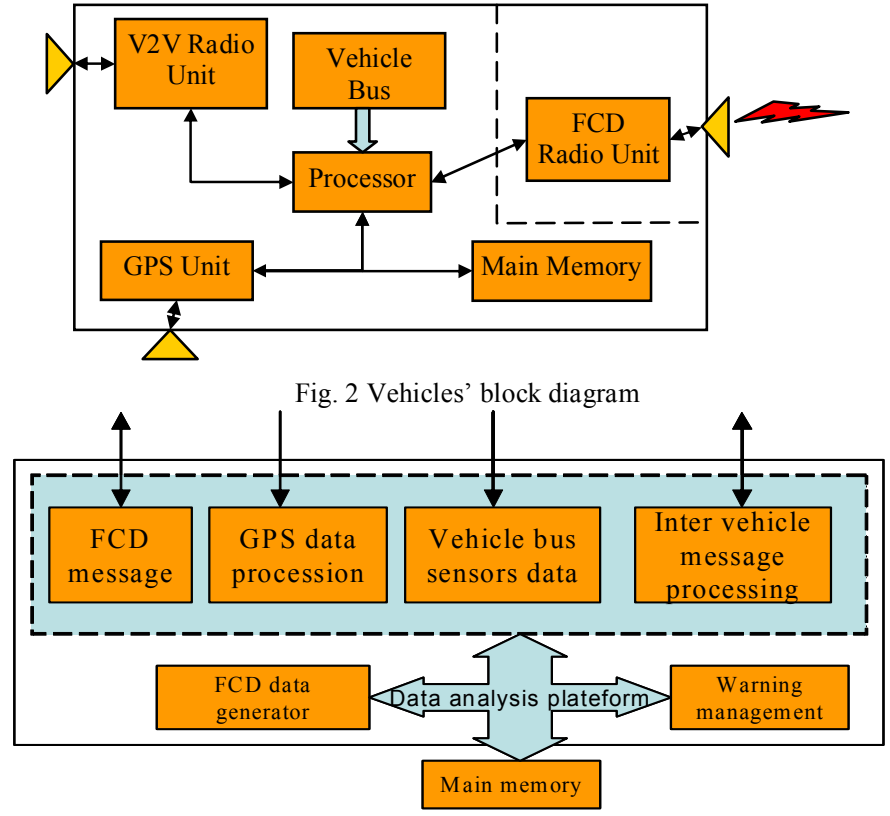

Fig. 3 Vehicle processor block diagram

Some V2V messages may be generated at the warning management module according to the implemented $\mathrm{V} 2 \mathrm{~V}$ applications. These warning messages are then sent out through the V2V radio unit. The same warning messages are added into the FCD data packet of the master-vehicle, which is transmitted to the FCD generator module together with the nearby floating vehicles data. In this FCD generator, the preliminary analysis of the raw data is carried out to eliminate some wrong data.

For example, at the normal centralized FCD system, after a map matching procedure, a part of the probe data will be deleted as useless data (e.g. vehicle is located outside of the road.) This process needs a very detailed digital map and matching algorithm. In our system, vehicle positions are projected positions, extrapolated from other local values. This position data is more reliable.

At the exit of the generator, some FCD packets are sent through FCD radio unit to the operating center, where a traffic information processor extracts the relevant information, like traffic jams and their positions. Some statistical analysis can be done for traffic prediction. Hardware/software solutions can be used to handle large amount of data. All this information is sent out to public using different Medias.

\section{COMMUNICATION SYSTEM REQUIREMENTS}

V2VC are made possible by the so-called Vehicular Ad-hoc NETworks (VANETs), a derivation of the more general self organized Mobile Ad-hoc NETworks (MANETs). In VANETs the high mobility and driving patterns of the nodes are the main constraints. An amendment of the IEEE 802.11 standard for the PHY/MAC layers, the 802.11p (also called WAVE: Wireless Access for Vehicular Environment), is under development, and it targets the specific requirements of VANET networks.

For active safety critical applications in the context of Intelligent Transport Systems (ITS), low latency and highly reliable transmissions are required. The transmissions used by these applications should be guaranteed to have highest priority to access the wireless media.

Broadcast routing protocols (controlled flooding) are mainly used locally in active safety critical applications. Unicast IP-based and/or location-based routing protocols are instead required by many other commercial and non-safety applications, to send or receive data to/from a specific geographic zone.

\section{A. Communication system description}

In our FCD-V2V application, some specific communication features need to be applied to realize the system requirements:

1) Application nature: this application is a non-safety application, implying that low latency or guaranteed priority are less critical. IP based routing can be used.

2) Network address auto-configuration: is the capability of network address automatic assignment to new nodes entering in the communication range, as well as to cancel the network address for the leaving nodes. Auto-configuration must handle situations like network partition and re-merging as well. It is a software solution, possibly coordinated by the master-vehicles.

3) Clustered-based systems. Around each master-vehicle, a clustered sub network is established between itself and the neighboring V2V-vehicles. The master vehicle should play the role of the cluster head of this sub network. The choice of the cluster-based network can be beneficial for the wireless traffic bandwidth to reduce channel contention. Fig. 4 shows a couple of examples of clusters.

\section{B. Networking}

1) Cluster head: to establish a cluster around the mastervehicle, an identifier-based clustering architecture is used. The master-vehicle transmits through the V2V unit periodic Hello messages, to announce its existence and to set to itself a specific identifier. This identifier will only be used for the cluster head. All of the V2V-vehicles entering the communication range of the master-vehicle are considered as a cluster member. Thanks to the auto-configuration function, a temporal network address is assigned to each cluster member. Special edge-of-cluster network management is required in order to maintain clusters well separated. The vehicles will choose the closest or best signal master-vehicle as its cluster head. As shown in Fig. 4, an overlapping of two cluster networks exists, node $\mathrm{C}$ and $\mathrm{D}$ can connect to both of these two cluster heads A and B. In this case, D will establish the connection only with the cluster A because of a shorter distance sector or better signal status. Moreover, in order to establish a relatively stable connectivity, a cluster member should intend to establish the connection with a cluster head moving in the same direction [6]. That is to say, node $\mathrm{C}$ will 
establish the connection with cluster head A instead of B, because $\mathrm{C}$ and $\mathrm{A}$ are moving to the same direction and the connection is more stable.

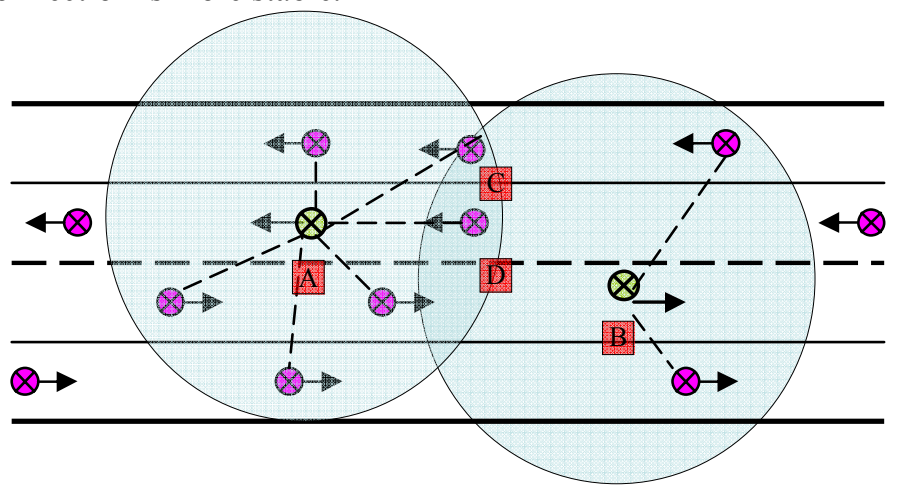

Fig. 4 Network description

A unicast (proactive) routing protocol is used for the FCD data transmission from and to the master-vehicle. Apparently in this application, there is no need to establish a connection with multiple cluster heads, namely, no Gateway function is necessary.

Once a connection with the cluster head node is established, it should remain as stable as possible, in order to keep a better availability for the data contribution. The fact of choosing identifier-based cluster is beneficial to the network stability [1].

2) Routing: It is both possible to implement multi-hop or one-hop routing methods. Multi-hop routing can help to improve the network scalability in the case of sparse network, but more control traffic will be needed for the routing procedure as well as the auto-configuration procedure.

\section{Up-link and down-link communications.}

So far we described a system where V2VC are used to extend the FCD data collection, and this is mainly helpful for the uplink data FCD transmission. As we know, traffic information obtained from FCD operation center can be diffused using different media to the public, such as GPRS, RDS-TMC, or the digital broadcasting (the latter fastly developing at the worldwide scale). In Beijing, a project of implementing DMB (Digital Media Broadcasting, a system derived from DAB (digital audio broadcasting, being able to carry the multimedia data.) has been programmed for the use of the traffic information diffusion. In our application, V2V systems can also be used for the information diffusion from master-vehicles to V2V-vehicles. See Fig. 3, the information received at the FCD radio unit will be transferred to the warning management module, therefore considered as warning messages and broadcasted to other $\mathrm{V} 2 \mathrm{~V}$-vehicles over the VANET, under the "traffic informing application".

\section{CONCLUSIONS AND FUTURE WORK}

The paper presents the conceptual architecture of a FCD system enforced by using V2V communication systems. Our goal is to improve the FCD application with a low cost, easily deployable method. In the proposed approach, the system is built upon the existing FCD infrastructure. By adding some networking features, both the reliability and quality of the traffic information are supposed to improve.

On the other hand, traffic efficiency applications in V2V/V2I systems can also be enforced in our architecture: besides the local V2VC-based warnings, vehicles would also be able to receive large scale traffic information through inter vehicle communications. Some applications that can not be realized at the local level, like dynamic navigation rerouting and near-future traffic prediction, will be available, even for the users who do not participate into the FCD project, who is in many cases limited by the marketing or political decision: individual vehicles will be able to contribute and benefit from the FCD systems.

The proposed architecture is still conceptual. The next step will be to prove its efficiency by simulations. In particular, the FCD uplink transmission will be supposed to be already available in the future simulation. The FCD data processing algorithm as well as the routing protocol concept based on the requirements will be the main challenges of the simulation. The actual status of our work is that, auto-configuration as well as the identifier-based cluster features have already been available in our team. Some stable unicast routing protocol based on the vehicle movement prediction is also undergoing.

\section{ACKNOWLEDGMENT}

Our gratitude is addressed to Mr. WenJia WANG from Hitachi China R\&D Cooperation, who has given us some very useful advices and explanations of our FCD related questions.

\section{REFERENCES}

[1] C. E. PERKINS, “AD HOC networking” Addiso-Wesley. U.S.A, vol. A370, pp. 75-135, December 2000.

[2] Car-to-Car Communication Consortium (C2C-CC), http://www.car-tocar.org.

[3] D.H. .859 "Vehicle Safety Communications Project: Task 3 final report, DOT". Annual report, Mar. 2005. [Online].

[4] Lars. Wischhof et al. "SOTIS - A self-Organizing traffic information system". FleetNet Project, German Ministry of Education and Research.

[5] Wenjia Wang et al. "A probe car system for Beijing". $12^{\text {th }}$ ITS world congress, San Francisco.

[6] H. Menouar, M. Lenardi, F. Filali, "A Movement Prediction based Routing Protocol for Vehicle-to-Vehicle Communications", V2 VCOM2005, July 2005, UCSD - La Jolla, CA, USA.

[7] Hans-J. Reumerman, Marco Roggero, Marco Ruffini, "The applicationbased clustering concept and requirements for intervehicle networks", IEEE Communication Magazine, April. 2005; EC Marie Curie Industry Host Fellowship contract - IST-2001-82950. 